\title{
An Observational Prospective Cohort Study of Incidence and Outcome of Streptococcus pneumoniae and Hemophilus influenzae Infections in Adult Solid Organ Transplant Recipients
}

\author{
Omid Rezahosseini ${ }^{1}\left(\mathbb{D}\right.$, Dina Leth Møller ${ }^{1}$, Søren Schwartz Sørensen ${ }^{2,3}$, Michael Perch ${ }^{3,4} \mathbb{D}$, Finn Gustafsson ${ }^{3,5}$, \\ Marco Gelpi ${ }^{1}$, Jenny Knudsen ${ }^{6}$ (D), Marie Helleberg ${ }^{7}$, Allan Rasmussen ${ }^{8}$, Susanne Dam Nielsen ${ }^{1,3}$ \\ and Zitta Barrella Harboe $1,9,10$,*
}

1 Viro-Immunology Research Unit, Department of Infectious Diseases, Rigshospitalet, University of Copenhagen, 2100 Copenhagen, Denmark; omid.rezahosseini@regionh.dk (O.R.); dina.leth.moeller@regionh.dk (D.L.M.); marco.gelpi@regionh.dk (M.G.); sdn@dadlnet.dk (S.D.N.)

2 Department of Nephrology, Rigshospitalet, University of Copenhagen, 2100 Copenhagen, Denmark; Soeren.Schwartz.Soerensen@regionh.dk

3 Department of Clinical Medicine, University of Copenhagen, 2100 Copenhagen, Denmark; Michael.Perch@regionh.dk (M.P.); Finn.Gustafsson@regionh.dk (F.G.)

check for updates

Citation: Rezahosseini, O.; Møller, D.L.; Sørensen, S.S.; Perch, M.; Gustafsson, F.; Gelpi, M.; Knudsen, J.; Helleberg, M.; Rasmussen, A.; Nielsen, S.D.; et al. An Observational Prospective Cohort Study of Incidence and Outcome of Streptococcus pneumoniae and Hemophilus influenzae Infections in Adult Solid Organ Transplant Recipients. Microorganisms 2021, 9 , 1371. https://doi.org/10.3390/ microorganisms 9071371

Academic Editor: Mathias W. Pletz

Received: 21 April 2021

Accepted: 22 June 2021

Published: 24 June 2021

Publisher's Note: MDPI stays neutral with regard to jurisdictional claims in published maps and institutional affiliations.

\section{Copyright: (c) 2021 by the authors.} Licensee MDPI, Basel, Switzerland. This article is an open access article distributed under the terms and conditions of the Creative Commons Attribution (CC BY) license (https:// creativecommons.org/licenses/by/ $4.0 /)$.
4 Section for Lung Transplantation, Department of Cardiology, Rigshospitalet, University of Copenhagen, 2100 Copenhagen, Denmark

5 Department of Cardiology, Rigshospitalet, University of Copenhagen, 2100 Copenhagen, Denmark

6 Department of Clinical Microbiology, Rigshospitalet, University of Copenhagen, 2100 Copenhagen, Denmark; Inge.Jenny.Dahl.Knudsen@regionh.dk

7 Centre of Excellence for Health, Immunity and Infections, Department of Infectious Diseases, Rigshospitalet, University of Copenhagen, 2100 Copenhagen, Denmark; Marie.Helleberg@regionh.dk

8 Department of Surgical Gastroenterology and Transplantation, Rigshospitalet, University of Copenhagen, 2100 Copenhagen, Denmark; Allan.Rasmussen@dadlnet.dk

9 Department of Bacteria, Parasites and Fungi, Statens Serum Institut, 2100 Copenhagen, Denmark

10 Department of Pulmonary and Infectious Diseases, Hospital of Nordsjælland, University of Copenhagen, 2100 Copenhagen, Denmark

* Correspondence: zitta.barrella.harboe@regionh.dk

\begin{abstract}
Background: Streptococcus pneumoniae (S. pneumoniae) and Hemophilus influenzae (H. influen$z a e$ ) are among the main vaccine-preventable bacterial infections in immunocompromised individuals including solid organ transplant (SOT) recipients. There is a lack of information about incidence and outcomes of these infections in SOT recipients. Methods: We determined the incidence of $S$. pneumoniae and H. influenzae, the related hospitalization, and 30- and 180-days mortality in a large cohort of 1182 adult SOT recipients. We calculated $95 \%$ confidence intervals (CI) of incidence rate (IR) using Byar's approximation to the Poisson distribution. Results: The overall IR of S. pneumoniae and H. influenzae were 1086 (95\% CI, 796-1448) and 1293 (95\% CI, 974-1687) per 100,000 person-years of follow-up (PYFU), respectively. The IR of invasive infections were 76 (95\% CI, 21-202) and 25 (95\% CI, 2.3-118) per 100,000 PYFU, respectively. Hospital admission was required in >50\%, 30-days mortality was 0 , and 180 -days mortality was $8.8 \%$ and $4.5 \%$ after S. pneumoniae and $H$. influenzae infections, respectively. Conclusions: The IR of invasive S. pneumoniae and H. influenzae infections in SOT recipients were much higher than reports from the general population in Denmark. Furthermore, a large proportion of infected SOT recipients were hospitalized. These findings highlight the need for further studies to assess uptake and immunogenicity of vaccines against S. pneumoniae and $H$. influenzae in SOT recipients.
\end{abstract}

Keywords: Streptococcus pneumoniae; invasive pneumococcal diseases; Hemophilus influenzae; organ transplant; incidence; hospitalization; mortality 


\section{Introduction}

Solid organ transplant (SOT) recipients receive life-long immunosuppression and therefore remain susceptible to infections that, in turn, lead to increased morbidity and mortality [1,2]. Etiology and incidence of infections vary according to the type of transplanted organ, time post-transplantation, and immunosuppression [3]. Streptococcus pneumoniae (S. pneumoniae) and Hemophilus influenzae (H. influenzae) are common bacterial causes of upper and lower respiratory tract infections and among the main vaccine-preventable bacterial infections post-transplantation [4,5]. Invasive infections, i.e., infections in normally sterile sites, are the most serious manifestations of infections caused by S. pneumoniae and $H$. influenzae with mortality rates as high as $29 \%$ in SOT recipients [6].

Studies reporting incidence rates (IR) of invasive and non-invasive S. pneumoniae and $H$. influenzae infections in SOT recipients are few [4]. However, vaccination against these bacteria is recommended based on data from studies conducted in pediatric SOT recipients, studies conducted prior to initiation of universal S. pneumoniae and H. influenzae vaccination programs, and studies in either immunocompetent or immunocompromised patients other than SOT recipients [4,5,7].

The aim of this study is to determine the incidence of S. pneumoniae and H. influenzae infections (invasive and non-invasive), the related hospitalization, and 30- and 180-days all-cause mortality in adult SOT recipients.

\section{Materials and Methods}

\subsection{Study Design and Participants}

In this observational prospective cohort study, we included adult ( $\geq 18$ years) SOT recipients (heart-, lung-, kidney-, liver-, simultaneous liver and kidney, and simultaneous kidney and pancreas recipients) who underwent SOT at Copenhagen University Hospital, Rigshospitalet, between 1 January 2011 and 31 December 2017. Due to the small number, combined liver and kidney transplant recipients were included in the group of liver transplant recipients. Combined pancreas and kidney transplant recipients were included as a separate group. Rigshospitalet is a highly specialized hospital that performs transplantations and the only center for liver and lung transplantation in Denmark. All SOT recipients were included in the Management of Posttransplant Infections in Collaborating Hospitals (MATCH) cohort. MATCH was established at Rigshospitalet in 2011 to improve management of infections post-transplantation [8].

We obtained clinical characteristics and microbiology data from the Centre of Excellence for Personalized Medicine of Infectious Complications in Immune Deficiency (PERSIMUNE) data warehouse [8]. Data were prospectively collected and merged as part of the routine care. Data from several clinical databases and national registries, such as the national Danish Microbiology Database (MiBa), are combined in the PERSIMUNE data repository. MiBa contains all microbiological data in Denmark from both hospitals and general practice since 2010 [9]. Data on S. pneumoniae and H. influenzae B vaccinations were not available.

The study was conducted in accordance with the declaration of Helsinki. The National Committee on Health Research Ethics (H-170024315) and the Data Protection Agency (04433, RH-2016-47) approved the retrieval of the data for the entire study. All experimental protocols were approved by the National Committee on Health Research Ethics of Denmark and the Danish Data Protection Agency. All relevant approval for this project was obtained from the Danish Health and Medicine Authorities. Informed consent for this type of study is not required according to national legislations. The National Committee on Health Research Ethics of Denmark waived the need for informed consent. All data was retrieved anonymously from the PERSIMUNE data repository and we did not have access to the medical records during the study therefore no further permissions were required. All methods were performed in accordance with the relevant guidelines and regulations. 


\subsection{Definitions}

Invasive infections were defined as positive cultures of S. pneumoniae and H. influenzae from normally sterile sites (blood, cerebrospinal fluid, synovial fluid, peritoneal fluid, and pleural fluid). Non-invasive infections were defined as positive cultures from non-sterile sites.

Among non-invasive infections, lower respiratory tract infections were defined as a positive cultures of $S$. pneumoniae and H. influenzae in bronchoalveolar lavage (BAL) or standard sputum specimens.

\subsection{Cultures}

All microbiological specimens were cultured according to the standard of care. The strain of $H$. influenzae was determined using matrix-assisted laser desorption ionizationtime of flight (MALDI-TOF) technique.

An episode of infection was defined as a positive culture from any site. In case of repeated positive cultures for a unique SOT recipient, we considered it as a new episode of infection, if the infection occurred more than 14 days from the previous episode of infection or if the bacterium was different [10].

\subsection{Follow-Up}

SOT recipients were followed from the date of transplantation to a positive culture, retransplantation, death, end of fifth year (day 1826) post-transplantation, or 31 December 2018 whichever came first. Last date of inclusion was 31 December 2017 to allow a period of follow-up for all SOT recipients. Infections that occurred after five years posttransplantation were not reported.

\subsection{Incidence of S. pneumoniae and H. influenzae Infections}

Number of cases and the IR of S. pneumoniae and H. influenzae infections (invasive and non-invasive) were reported for the entire follow-up period. We reported the overall IR of S. pneumoniae and H. influenzae infections, the IR of invasive infections, and the IR according to the type of transplanted organ.

\subsection{Statistical Analysis}

Proportions were reported as percentage, and continuous data were reported as medians with interquartile ranges (IQR). Mann-Whitney U test was used to compare the differences in medians, and Fisher's exact test was used to test the frequency distributions. We calculated 95\% confidence intervals (CI) of IR using Byar's approximation to the Poisson distribution. Estimates of the cumulative incidence of the first episode of S. pneumoniae and $H$. influenzae infection were calculated using Aalen-Johansen estimator with death and retransplantation as competing risks. Statistical differences were tested using Grey's test. In two explorative analysis, we conducted a matched case-control study to compare all-cause mortality at any time after transplantation in SOT recipients who had and did not have S. pneumoniae or $H$. influenzae infection. SOT recipients with positive infection were assumed as cases whereas controls were selected from SOT recipients who did not have infection. Cases and controls matched on age, gender, the type of transplantation, and days post-transplantation. Fisher's exact test was used to test all-cause mortality between cases and controls. MatchIt package was used for the case-control part using the nearest neighbor-matching method and 1:3 ratio. $\mathrm{R}$ software version 3.5.2 was used for statistical analyses, and a $p$ value $\leq 0.05$ was considered statistically significant.

\section{Results}

\subsection{Patient Characteristics}

We included 1182 adults SOT recipients; 577 (49\%) kidney, 293 (25\%) liver, 210 (18\%) lung, 84 (7.1\%) heart, and 18 (1.5\%) simultaneous pancreas and kidney transplant recipients. Among these, $726(61 \%)$ were male and median age at the time of transplantation was 50 (IQR 41-59) years. 
Applying criteria for follow-up that we mentioned in the methods, 24 (2\%) SOT recipients were re-transplanted and $169(14 \%)$ died. The median time to retransplantation was 223 (IQR 94-667) days and for the death was 445 (IQR 170-938) days post-transplantation.

SOT recipients had a median of 23 (IQR 8-54) cultures from any site and for any reason. Only $48(4 \%)$ of 1182 SOT recipients did not have any cultures performed during follow-up.

\subsection{S. pneumoniae Infection}

Forty-three episodes of S. pneumoniae infections were found in 34 SOT recipients. Four of $34(12 \%)$ SOT recipients had more than one episode. Total of $3(7 \%), 35(81 \%)$, and $5(12 \%)$ of 43 episodes were found in blood, lower respiratory tract, and other sites, respectively. The three blood stream infections were the only invasive infections. Patient characteristics of SOT recipients with and without at least one episode of $S$. pneumoniae infection are shown in Table 1. All three SOT recipients with invasive S. pneumoniae infection and $15(48 \%)$ of 31 SOT recipients with non-invasive $S$. pneumoniae infection were admitted to hospital. None $(0 \%)$ and $3(8.8 \%)$ of SOT recipients with S. pneumoniae infection died (all-cause) during 30-days and 180-days of follow-up, respectively. In a matched case-control, we did not observe statistically significant difference between all-cause mortality at any time after transplantation in SOT recipients who had and did not have S. pneumoniae infection (Supplementary Table S1).

Table 1. Characteristics of solid organ transplant recipients who had and did not have at least one episode of S. pneumoniae infection.

\begin{tabular}{|c|c|c|c|c|c|}
\hline \multicolumn{2}{|c|}{ Characteristics } & $\begin{array}{l}\text { At Least One Positive } \\
\text { Culture for } \\
\text { S. pneumoniae }(\mathrm{n}=34)\end{array}$ & $\begin{array}{l}\text { No Positive Culture for } \\
\text { S. pneumoniae } \\
(\mathrm{n}=1148)\end{array}$ & Total $(n=1182)$ & $p$-Value \\
\hline \multirow{2}{*}{\multicolumn{2}{|c|}{$\begin{array}{l}\text { Age, median (IQR) } \\
\text { Male (n, \%) }\end{array}$}} & $51(38,58)$ & $50(41,59)$ & $50(41,59)$ & 0.63 \\
\hline & & $22(65)$ & $704(61)$ & $726(61)$ & 0.73 \\
\hline \multirow{5}{*}{$\begin{array}{c}\text { Transplanted } \\
\text { organ }(n, \%)\end{array}$} & Heart & $3(8.8)$ & $81(7.1)$ & $84(7.1)$ & \multirow{5}{*}{0.90} \\
\hline & Liver & $10(29)$ & $283(25)$ & $293(25)$ & \\
\hline & Lung & $6(18)$ & $204(18)$ & $210(18)$ & \\
\hline & Kidney & $15(44)$ & $562(49)$ & $577(49)$ & \\
\hline & $\begin{array}{c}\text { Simultaneous } \\
\text { pancreas/kidney }\end{array}$ & $0(0.0)$ & 18 (1.6) & $18(1.5)$ & \\
\hline
\end{tabular}

\subsection{Incidence Rates of S. pneumoniae Infection}

In analyses of S. pneumoniae infections there were 3961 person-years of follow-up (PYFU). The overall IR of S. pneumoniae infection (invasive and non-invasive) during the first five years post-transplantation was 1086 per 100,000 PYFU (95\% CI, 796-1448). The IR of invasive S. pneumoniae infection was 76 per 100,000 PYFU (95\% CI, 21-202) (Table 2).

The overall IRs of S. pneumoniae infection according to type of transplanted organ are shown in Table 2.

The cumulative incidence of the first episode of S. pneumoniae infection in the first five years post-transplantation was $3.9 \%(95 \% \mathrm{CI}, 0.63-7.2)$. There were no significant differences in cumulative incidence of $S$. pneumoniae infection according to the type of transplanted organ $(p=0.82)$ (Figure 1A). 
Table 2. Incidence rates of S. pneumoniae and H. influenzae infections.

\begin{tabular}{|c|c|c|c|}
\hline Category ( $N$ = Population Size) & $\begin{array}{c}\text { Time Interval } \\
\text { Post-Transplantation }\end{array}$ & $\begin{array}{l}\text { Incidence Rate }(95 \% \mathrm{CI}) \text { of } S . \\
\text { pneumoniae per } 100,000 \text { PYFU }\end{array}$ & $\begin{array}{c}\text { Incidence Rate }(95 \% \mathrm{CI}) \text { of } H \text {. } \\
\text { influenzae per } 100,000 \text { PYFU }\end{array}$ \\
\hline \multirow{4}{*}{$\begin{array}{l}\text { Overall (invasive and non-invasive } \\
\text { infections) } N=1182\end{array}$} & 5 years & $1086(796-1448)$ & $1293(974-1687)$ \\
\hline & First year & 1235 (707-2017) & 1059 (578-1794) \\
\hline & First six months & $873(331-1914)$ & $1049(436-2163)$ \\
\hline & First month & $1000(91-4662)$ & $1002(91-4672)$ \\
\hline \multirow{2}{*}{ Only invasive infection $N=1182$} & 5 years & $76(21-202)$ & $25(2-118)$ \\
\hline & First year & $88(8-411)$ & $88(8-411)$ \\
\hline Heart transplant recipients $N=84$ & 5 years & $1682(638-3688)$ & $33(3-156)$ \\
\hline Lung transplant recipients $N=210$ & 5 years & $996(414-2053)$ & 1517 (749-2770) \\
\hline \multirow{2}{*}{ Liver transplant recipients $N=293$} & 5 years & $1410(790-2343)$ & $1091(560-1935)$ \\
\hline & $\begin{array}{l}\text { Only invasive } \\
\text { infection, } 5 \text { years }\end{array}$ & $217(43-695)$ & $109(10-509)$ \\
\hline \multirow{2}{*}{ Kidney transplant recipients $N=577$} & 5 years & $903(562-1381)$ & $1477(1023-2069)$ \\
\hline & $\begin{array}{l}\text { Only invasive } \\
\text { infection, } 5 \text { years }\end{array}$ & $48(4-221)$ & $-*$ \\
\hline $\begin{array}{l}\text { Simultaneous pancreas and kidney } \\
\text { transplant recipients } N=18\end{array}$ & 5 years & $-*$ & $-*$ \\
\hline
\end{tabular}

* No infection was reported in this group.

\subsection{H. influenzae Infection}

There were 51 episodes of $H$. influenzae infection in 44 SOT recipients. Three $(6.8 \%)$ of 44 SOT recipients had more than one episode. Total of $1(2 \%), 45(88 \%)$, and $5(10 \%)$ of 51 episodes were found in blood, lower respiratory tract, and other sites, respectively. We found three SOT recipients who had simultaneous positive cultures for both $\mathrm{H}$. influenzae and S. pneumoniae from lower respiratory tract. Patient characteristics of SOT recipients with and without at least one episode of $H$. influenzae infection are shown in Table 3 . The SOT recipient with invasive $H$. influenzae infection was not admitted to hospital, but 22 of $43(51 \%)$ SOT recipients with non-invasive $H$. influenzae infection were admitted to hospital. None of 44 SOT recipients with $H$. influenzae infection died during 30-days, but 2 (4.5\%) died (all-cause) during 180-days of follow-up after $H$. influenzae infection, none of them had invasive infection. In a matched case-control, we did not observe statistically significant difference between all-cause mortality at any time after transplantation in SOT recipients who had and did not have H. influenzae infection (Supplementary Table S2).

\subsection{Incidence Rates of H. influenzae Infection}

In analyses of $H$. influenzae infections there were 3941 PYFU. The overall IR of $H$. influen$z a e$ infection (invasive and non-invasive) during the first five years post-transplantation was 1293 per 100,000 PYFU (95\% CI, 974-1687). The IR of invasive H. influenzae infection was 25 per 100,000 PYFU (95\% CI, 2-118) (Table 2).

The overall IRs of $H$. influenzae infection according to the type of transplanted organ are shown in Table 2. The cumulative incidence of H. Influenzae infection in the first five years post-transplantation was $5.0 \%(95 \% \mathrm{CI}, 1.8-8.1)$. There were no significant differences in cumulative incidence of $H$. influenzae infection according to the type of transplanted organ $(p=0.43)$ (Figure 1B). 
A

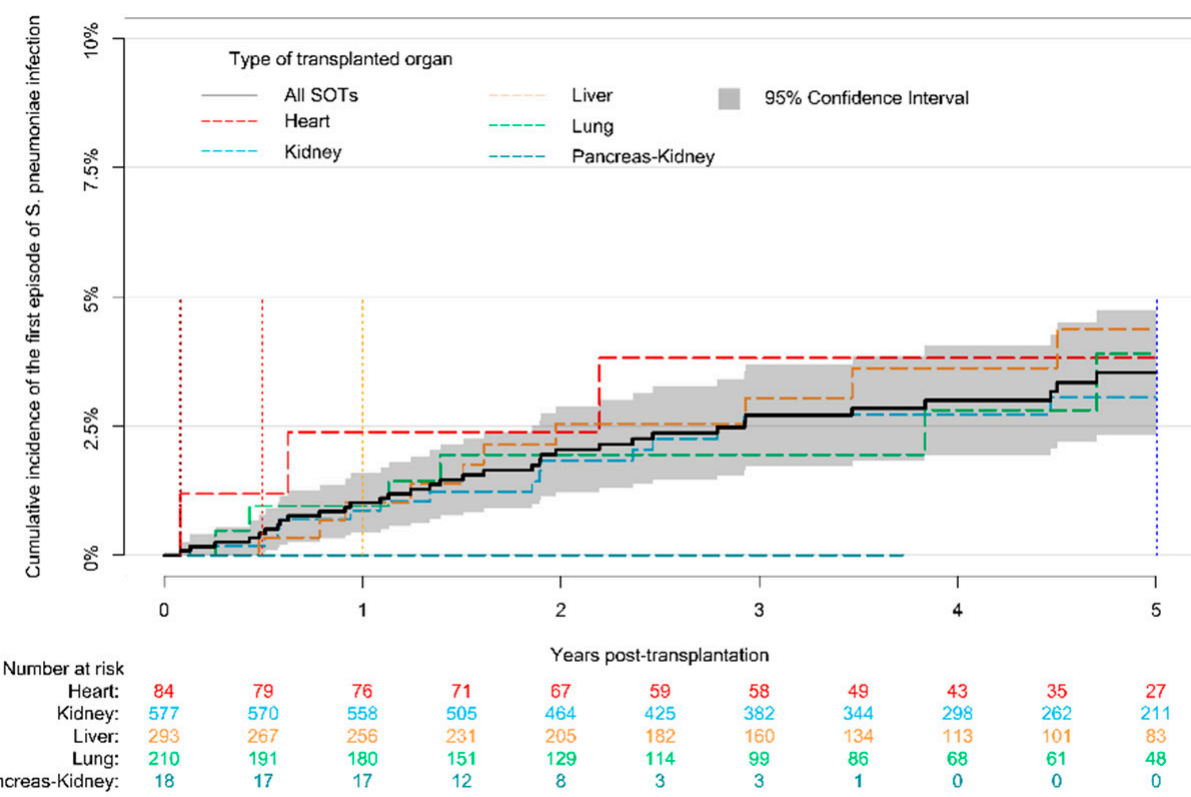

B

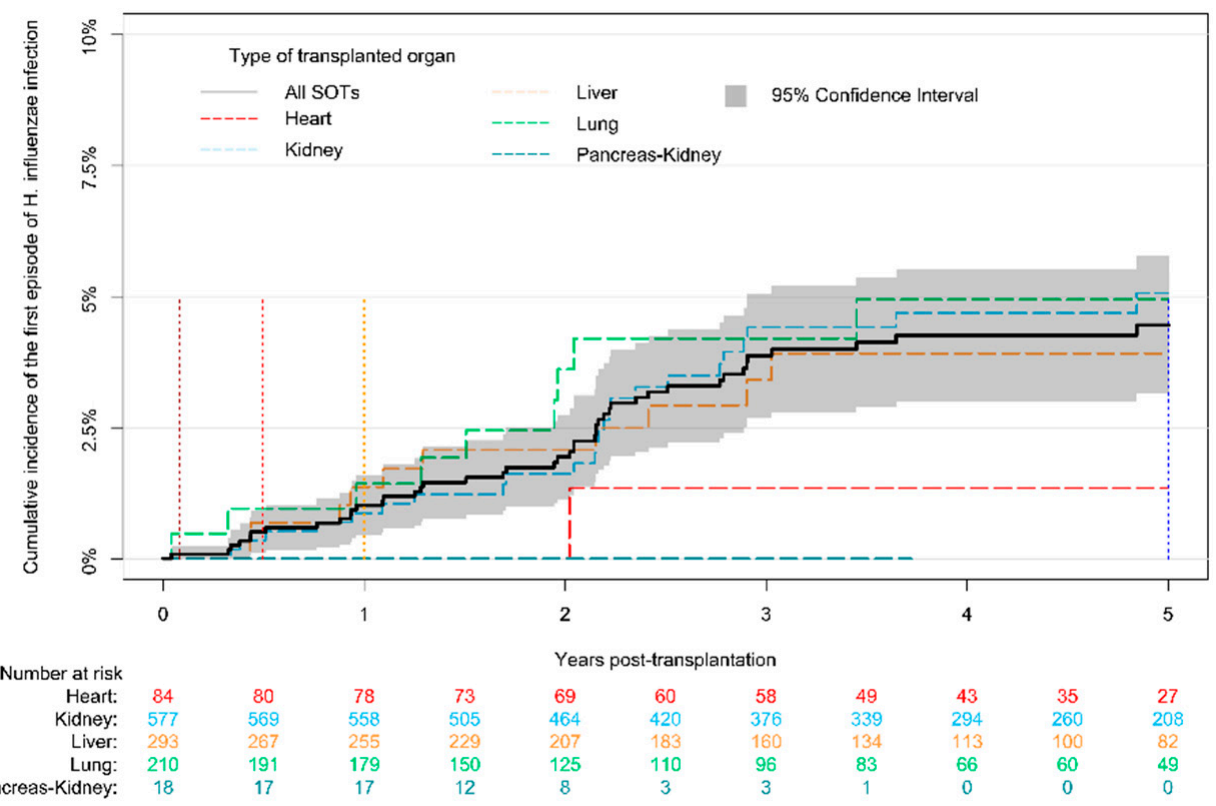

Figure 1. Cumulative incidence of the first episode of S. pneumoniae and H. influenzae infections during the five years post-transplantation. (A) Cumulative incidence (3.9\% (95\% CI, 0.63-7.2)) of the first episode of $S$. pneumoniae infection. There was no statistically significant difference in cumulative incidence of the first episode of S. pneumoniae infection between different types of transplanted organs ( $p=0.82)$. (B) Cumulative incidence (5.0\% (95\% CI, 1.8-8.1)) of the first episode of $H$. influenzae infection. There was no statistically significant difference in cumulative incidence of the first episode of $H$. influenzae infection between different types of transplanted organs $(p=0.57)$. Simultaneous kidney and pancreas transplant recipients had no episode of $H$. influenzae infection. Black continuous line shows the cumulative incidence of the first episode of infection in the total cohort of solid organ transplant (SOT) recipients. Colorful dashed lines indicate cumulative incidence of the first episode of infection according to the type of transplanted organ. Vertical dotted-lines determine 30 days (brown), 6 months (red), 1 year (yellow), and 5 years (blue) post-transplantation. 
Table 3. Characteristics of solid organ transplant recipients who had and did not have at least one episode of H. influenzae infection.

\begin{tabular}{|c|c|c|c|c|c|}
\hline \multicolumn{2}{|c|}{ Characteristics } & $\begin{array}{c}\text { At Least One Positive } \\
\text { Culture for } H \text {. } \\
\text { influenzae }(\mathrm{n}=44)\end{array}$ & $\begin{array}{l}\text { No Positive Culture for } \\
\text { H. influenzae }(\mathrm{n}=1138)\end{array}$ & Total $(\mathrm{n}=\mathbf{1 1 8 2})$ & $p$-Value \\
\hline \multirow{2}{*}{\multicolumn{2}{|c|}{$\begin{array}{l}\text { Age, median (IQR) } \\
\text { Male }(n, \%)\end{array}$}} & $55(46,60)$ & $50(41,59)$ & $50(41,59)$ & 0.10 \\
\hline & & $26(59)$ & $700(62)$ & $726(61)$ & 0.75 \\
\hline \multirow{5}{*}{$\begin{array}{l}\text { Transplanted } \\
\text { organ }(n, \%)\end{array}$} & Heart & $1(2.3)$ & $83(7.3)$ & $84(7.1)$ & \\
\hline & Liver & $10(23)$ & $283(25)$ & $293(25)$ & \\
\hline & Lung & $9(21)$ & $201(18)$ & $210(18)$ & 0.73 \\
\hline & Kidney & $24(55)$ & $553(49)$ & 577 (49) & \\
\hline & $\begin{array}{c}\text { Simultaneous } \\
\text { pancreas/kidney }\end{array}$ & $0(0.0)$ & $18(1.6)$ & $18(1.5)$ & \\
\hline
\end{tabular}

\section{Discussion}

In this large, prospective study of adult SOT recipients with complete follow-up and nationwide data on all microbiology from both hospitals and general practice, we found that both the overall IR of S. pneumoniae and H. influenzae infections and IR of invasive infections were considerable. All-cause mortality was, $8.8 \%$ and $4.5 \%$ during 180 days of follow-up after S. pneumoniae and H. influenzae infections, respectively.

In a cohort study that was done between 2001 and 2014, the IR of IPD was 414 per 100,000 PYFU [11]. In a recent meta-analysis, the pooled IR of invasive S. pneumoniae infections in SOT recipients was 465 per 100,000 PYFU [11] which is about six times higher than our estimates. These studies also included patients from before 2007 when vaccination against $S$. pneumoniae was not yet included in the universal childhood vaccination programs which may explain the discrepancy [11,12]. The 7-valent pneumococcal conjugated vaccine was introduced in Europe in 2001 and in the Danish Childhood Vaccination Program in 2007 [13,14]. Moreover, from October 2012, a limited subsidy has been introduced by the Danish Health and Medicines Authority for pneumococcal vaccination in highrisk adults [15]. The IR of invasive S. pneumoniae infections in the 38 to 60 years old general population in Denmark decreased from 14 per 100,000 person-years to 10 per 100,000 PYFU during the period 2011 to 2017, possibly as a result of herd immunity and sporadic vaccinations in adults (Unpublished data from Statens Serum Institut (SSI)). It is likely that SOT recipients in Denmark also have benefitted from this indirect effect of childhood vaccination or sporadically got vaccinated. Furthermore, although a routine program for vaccination of all SOT candidates for liver, lung, heart, and pancreas only was introduced in 2020, it is likely that some of the SOT recipients in our study were vaccinated against S. pneumoniae prior to or after transplantation. However, a recent study from Denmark showed that the coverage of vaccination against S. pneumoniae in the kidney transplant recipients was only 4\% [16]. Moreover, according to unpublished data from Knowledge Center of Transplantation at Rigshospitalet, only $51(18 \%)$ and $2(0.7 \%)$ out of 281 liver transplant recipients who were transplanted from 1 January 2011 to 31 December 2017 had a documented positive history of vaccination against $S$. pneumoniae and H. influenzae B, respectively (Unpublished data).

We found a subpopulation of culture positive SOT recipients who had more than one episode of infection with S. pneumoniae (12\%) and H. influenzae (6.8\%). It is possible that this subpopulation of SOT recipients received more intensive immunosuppression, but we did not have access to the medication history to confirm this.

The IR of invasive H. influenzae infection in adult SOT recipients has not previously been reported. We found the IR of invasive H. Influenzae infection was 25 per 100,000 PYFU. For comparison, the mean IR of invasive H. influenzae was 1.3 (95\% CI 1.1 to 1.5) per 100,000 PYFU in the 38 to 60 years old general population of Denmark in the period 2011-2017 (unpublished data from SSI). 
All three SOT recipients with invasive S. pneumoniae infection as well as half of the recipients with either non-invasive $S$. pneumoniae or $H$. influenzae infections were admitted to the hospital. The in-hospital mortality after invasive S. pneumoniae infection in SOT recipients has previously been reported to be as high as $29 \%$ in a study from Canada [6] and the overall mortality in general population within the 28 days after invasive $H$. influenzae infection was $9.8 \%$ in a study from Sweden [17]. In our cohort there were no deaths within 30 -days, but $8.8 \%$ and $4.5 \%$ of SOT recipients died from all causes during 180 -days after S. pneumoniae and $H$. influenzae infections, respectively. Vaccination and herd immunity against $S$. pneumoniae infection, higher awareness about invasive infections and rapid initiation of antibiotics could be the possible explanations for this low mortality. Although, in matched case-control analysis, mortality at any time after transplantation was not different between SOT recipients who had and did not have infection.

The large sample size with complete follow-up, prospective gathering of data, and nationwide data on microbiological tests were the strengths of our study. However, we did not have access to serotype of isolates, immunosuppressive drugs, or vaccination history. Thus, we are unable to determine the effect of these parameters on the incidence of $S$. pneumoniae and $H$. influenzae infections. Moreover, only a few liver and kidney transplant recipients were routinely vaccinated prior to 2020. As such, our data may overestimate the magnitude of infections in a fully vaccinated cohort. In Denmark, the H. influenzae is always identified using MALDI-TOF. The S. pneumoniae can be identified using MALDITOF, but in most cases is identified using the optochin test (optochin susceptible and catalase negative). Only $H$. influenzae type B is vaccine preventable and most of the cases of community-acquired pneumonia, otitis media, and exacerbations of chronic obstructive pulmonary disease are caused by non-typable (i.e., non-vaccine preventable) strains of $H$. influenzae [18]. Unfortunately, we were not able to provide information about the strains of the $S$. pneumoniae and $H$. influenzae which is a limitation to the study.

\section{Conclusions}

The IR of invasive S. pneumoniae and H. influenzae infections in SOT recipients is much higher than that reported in the general population in Denmark. However, mortality of $S$. pneumoniae and $H$. influenzae infections was lower than expected, albeit a large proportion of infected SOT recipients are hospitalized. S. pneumoniae and $H$. influenzae are vaccine preventable diseases, and further studies to assess the uptake and immunogenicity of vaccines against $S$. pneumoniae and $H$. influenzae in SOT recipients are warranted.

Supplementary Materials: The following are available online at https:/ /www.mdpi.com/article/10.3 390/microorganisms9071371/s1, Supplementary Table S1: Characteristics of the matched cases and controls who had and did not have S. pneumoniae infection, Supplementary Table S2: Characteristics of the matched cases and controls who had and did not have H. influenzae infection.

Author Contributions: O.R., D.L.M., S.S.S., M.P., F.G., M.G., J.K., M.H., A.R., S.D.N. and Z.B.H. designed the study. All authors collected the data. O.R., S.D.N. and Z.B.H. did statistical analyses. J.K. managed the laboratory tests. O.R., S.D.N. and Z.B.H. wrote the manuscript. All authors have read and agreed to the published version of the manuscript.

Funding: This work was supported by the Novo Nordisk Foundation, Independent Research Fund (FSS), the Danish National Research Foundation (grant number DNRF126), and the Research Foundation of Rigshospitalet.

Institutional Review Board Statement: The study was conducted according to the guidelines of the Declaration of Helsinki, and approved by the National Committee on Health Research Ethics (H-170024315) and the Data Protection Agency (04433, RH-2016-47).

Informed Consent Statement: Patient consent was waived according to national legislations. The National Committee on Health Research Ethics of Denmark waived the need for informed consent. All data was retrieved anonymously from the PERSIMUNE data repository and we did not have access to the medical records during the study therefore no further permissions were required. 
Data Availability Statement: The data presented in this study are available on request from the corresponding author. The data are not publicly available due to Danish legislations.

Acknowledgments: We sincerely thank all SOT recipients as well as staff at MATCH and PERSIMUNE for their contribution to this study. We also thank Tine Dalby, MSc, PhD, for providing data from the National Laboratory Surveillance for S. pneumoniae and H. influenzae at SSI of Denmark. Moreover, we thank Sebastian Rask Hamm, MBBS, for collecting information about S. pneumoniae and $H$. influenzae vaccination in liver transplant recipients.

Conflicts of Interest: O.R. received a grant from The Research Foundation of Rigshospitalet; S.S.S. reported other non-financial relations with Alexion, Hansa Biopharma, and Corline Biomedical, outside the submitted work; M.P. received a grant from Roche, non-financial support from Boehringer Ingelheim, personal fees from Mallinckrodt, Novartis, GSK, and Astra-Zeneca not related to this work; F.G. received personal fees from Abbott, Novartis, Boehringer-Ingelheim, Orion Pharma, Pfizer, and AstraZeneca and had other financial relationship with Corvia not related to this work; M.G. received a grant from Augustinus Foundation not related to this work; S.D.N. received unrestricted research grants from Novo Nordisk Foundation and Independent Research Fund (FSS); D.L.M., J.K., M.H., A.R. and Z.B.H. declare no conflicts of interest. The funders had no role in the design of the study; in the collection, analyses, or interpretation of data; in the writing of the manuscript, or in the decision to publish the results.

\section{References}

1. Van Delden, C.; Stampf, S.; Hirsch, H.H.; Manuel, O.; Meylan, P.; Cusini, A.; Hirzel, C.; Khanna, N.; Weisser, M.; Garzoni, C.; et al. Burden and timeline of infectious diseases in the first year after solid organ transplantation in the swiss transplant cohort study. Clin. Infect. Dis. 2020, 71, e159-e169. [CrossRef] [PubMed]

2. Fishman, J.A. Infection in Organ Transplantation. Am. J. Transplant. 2017, 17, 856-879. [CrossRef] [PubMed]

3. Singh, N.; Haidar, G.; Limaye, A.P. Infections in Solid-Organ Transplant Recipients. In Mandell, Douglas, and Bennett's Principles and Practice of Infectious Diseases; Bennett, M.D., Dolin, J.E., Blaser, R., Eds.; Elsevier: Hoboken, NJ, USA, 2020; pp. 3672-3697.e4. ISBN 978-0-323-48255-4 BT.

4. Danziger-Isakov, L.; Kumar, D. Vaccination of solid organ transplant candidates and recipients: Guidelines from the American society of transplantation infectious diseases community of practice. Clin. Transplant. 2019, 33, e13563. [CrossRef]

5. Dendle, C.; Stuart, R.L.; Mulley, W.R.; Holdsworth, S.R. Pneumococcal vaccination in adult solid organ transplant recipients: A review of current evidence. Vaccine 2018, 36, 6253-6261. [CrossRef]

6. Kumar, D.; Humar, A.; Plevneshi, A.; Green, K.; Prasad, G.V.R.; Siegal, D.; McGeer, A. Invasive pneumococcal disease in solid organ transplant recipients-10-year prospective population surveillance. Am. J. Transplant. Off. J. Am. Soc. Transplant. Am. Soc. Transpl. Surg. 2007, 7, 1209-1214. [CrossRef] [PubMed]

7. Rubin, L.G.; Levin, M.J.; Ljungman, P.; Davies, E.G.; Avery, R.; Tomblyn, M.; Bousvaros, A.; Dhanireddy, S.; Sung, L.; Keyserling, H.; et al. 2013 IDSA clinical practice guideline for vaccination of the immunocompromised host. Clin. Infect. Dis. 2014, 58, e44-e100. [CrossRef] [PubMed]

8. Centre of Excellence for Personalized Medicine of Infectious Complications in Immune Deficiency (PERSIMUNE). Available online: http:/ / www.persimune.dk/ (accessed on 19 November 2020).

9. Voldstedlund, M.; Haarh, M.; Mølbak, K. The danish microbiology database (MIBA) 2010 to 2013. Eurosurveillance 2014, 19 , 20667. [CrossRef] [PubMed]

10. Centers for Disease Control and Prevention Identifying Healthcare-Associated Infections (HAI) for NHSN Surveillance. Available online: https://www.cdc.gov/nhsn/PDFs/pscManual/2PSC_IdentifyingHAIs_NHSNcurrent.pdf (accessed on 19 November 2020).

11. Mahmud, S.M.; Sinnock, H.; Mostaço-Guidolin, L.C.; Pabla, G.; Wierzbowski, A.K.; Bozat-Emre, S. Long-term trends in invasive pneumococcal disease in Manitoba, Canada. Hum. Vaccin. Immunother. 2017, 13, 1884-1891. [CrossRef] [PubMed]

12. van Aalst, M.; Lötsch, F.; Spijker, R.; van der Meer, J.T.M.; Langendam, M.W.; Goorhuis, A.; Grobusch, M.P.; de Bree, G.J. Incidence of invasive pneumococcal disease in immunocompromised patients: A systematic review and meta-analysis. Travel. Med. Infect. Dis. 2018, 24, 89-100. [CrossRef] [PubMed]

13. De Carvalho Gomes, H.; Muscat, M.; Monnet, D.L.; Giesecke, J.; Lopalco, P.L. Use of seven-valent pneumococcal conjugate vaccine (PCV7) in Europe, 2001-2007. Euro Surveill. Bull. Eur. Mal. Transm. Eur. Commun. Dis. Bull. 2009, 14, 19159.

14. Statens Serum Institut Invasive Pneumococcal Disease 2018-2019. Available online: https://en.ssi.dk/surveillance-andpreparedness / surveillance-in-denmark/annual-reports-on-disease-incidence/invasive-pneumococcal-disease-2018-2019 (accessed on 15 October 2020).

15. Pneumococcal Vaccination of Persons at Increased Risk of Invasive Pneumococcal Disease. Statens Serum Institut. Available online: https: / / en.ssi.dk/news/epi-news/2012/no-51b---2012 (accessed on 29 May 2021).

16. Larsen, L.; Bistrup, C.; Sørensen, S.S.; Boesby, L.; Nguyen, M.T.T.; Johansen, I.S. The coverage of influenza and pneumococcal vaccination among kidney transplant recipients and waiting list patients: A cross-sectional survey in Denmark. Transpl. Infect. Dis. 2020, e13537. [CrossRef] 
17. Resman, F.; Ristovski, M.; Ahl, J.; Forsgren, A.; Gilsdorf, J.R.; Jasir, A.; Kaijser, B.; Kronvall, G.; Riesbeck, K. Invasive disease caused by Haemophilus influenzae in Sweden 1997-2009; evidence of increasing incidence and clinical burden of non-type b strains. Clin. Microbiol. Infect. Off. Publ. Eur. Soc. Clin. Microbiol. Infect. Dis. 2011, 17, 1638-1645. [CrossRef] [PubMed]

18. Bakaletz, L.O.; Novotny, L.A. Nontypeable Haemophilus influenzae (NTHi). Trends Microbiol. 2018, 26, 727-728. [CrossRef] [PubMed] 